MATHEMATICS OF COMPUTATION

Volume 72 , Number 242 , Pages 657-675

S 0025-5718(02)01456-4

Article electronically published on June 4, 2002

\title{
THEORETICAL AND NUMERICAL ANALYSIS FOR THE QUASI-CONTINUUM APPROXIMATION OF A MATERIAL PARTICLE MODEL
}

\author{
PING LIN
}

\begin{abstract}
In many applications materials are modeled by a large number of particles (or atoms) where any one of particles interacts with all others. Near or nearest neighbor interaction is expected to be a good simplification of the full interaction in the engineering community. In this paper we shall analyze the approximate error between the solution of the simplified problem and that of the full-interaction problem so as to answer the question mathematically for a one-dimensional model. A few numerical methods have been designed in the engineering literature for the simplified model. Recently much attention has been paid to a finite-element-like quasicontinuum (QC) method which utilizes a mixed atomistic/continuum approximation model. No numerical analysis has been done yet. In the paper we shall estimate the error of the QC method for this one-dimensional model. Possible ill-posedness of the method and its modification are discussed as well.
\end{abstract}

\section{INTRODUCTION}

The analysis of the structure of material defects such as dislocations or fractures has to consider the effects at the lattice scale. Directly solving the whole system (e.g., in the lattice statics and the molecular dynamics model) provides a powerful and accurate tool of analysis at this scale. However, because the number of particles (or atoms) in a material is huge, it is often impossible to solve the whole system with present-day computers. The problem is often simplified by only considering the interaction of one particle with its nearby particles (or even its nearest neighbors). It is believed that the simplified problem is a good approximation to the original problem. But there is no mathematical proof available. On the other hand, even for the simplified problem the system is still huge and impossible to solve directly. Recently a method called quasicontinuum (QC) approximation has gained noticeable attention in the engineering literature (cf. [4, 1, 7]). The idea is that in the region where no defect occurs the material is modeled at the macroscopic scale and the theory of continuum elasticity may apply. It is incorporated with the finite element method and is expected to be an approximation of the full latticescale model. Numerical analysis of this approximation method is in its infancy.

Received by the editor June 9, 1998 and, in revised form, May 29, 2001.

2000 Mathematics Subject Classification. Primary 65C20, 65K10, 65M15, 65M60, 74N15, $74 \mathrm{G} 65$.

Key words and phrases. Lattice statics, particle motion, Lennard-Jones potential, global minimization, finite element method, error estimation, ill-posedness, quasi-continuum approximation, material modeling. 

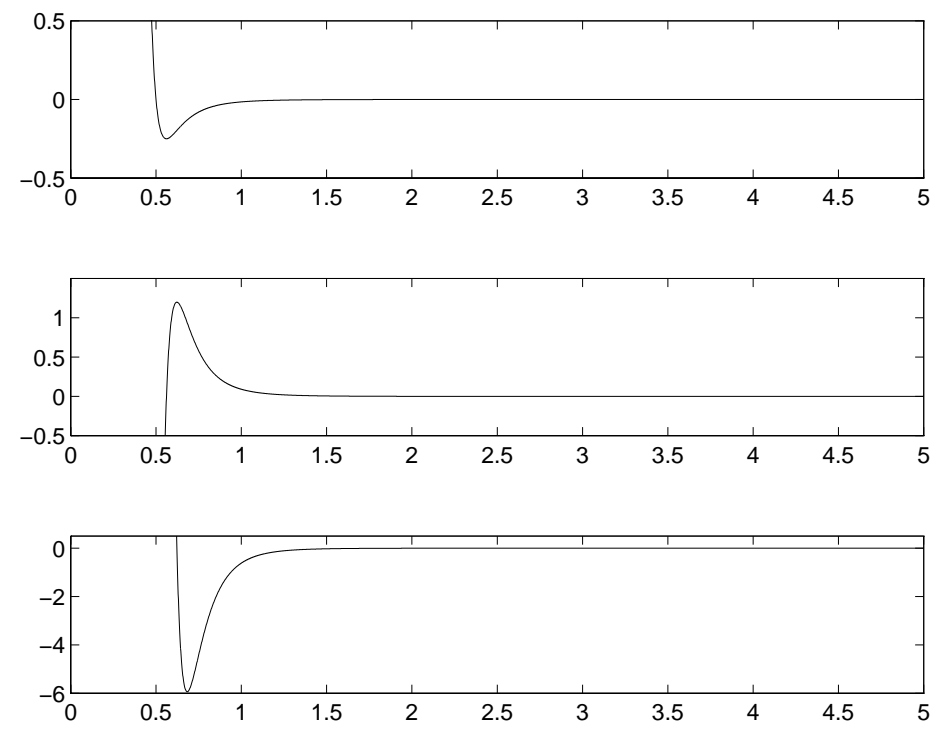

Figure 1. The functions $W(\alpha), W^{\prime}(\alpha)$ and $W^{\prime \prime}(\alpha)$ with $\sigma=0.5$

The model is a system incorporating a large number of material particles. Unlike typical physical problems, the discrete subdomain of the approximation method is treated not as one small body but as a composition of a number of particles. As pointed out in [5], the presence of microstructures has motivated the development of numerical methods that can capture macroscopic information without resolving the microstructure on the physical length scale. The QC method seems to be such an example. As an initial theoretical study of the QC method we consider a typical one-dimensional crystal material (an atomistic chain where interacting energy of any two atoms is the Lennard-Jones potential [3, 6]). Study of similar models can also be found in [2] and in [6], with emphasis on wave propagation and on nearest neighbor interaction under simpler potential functions which lead to a linearization of the mathematical problem. In this paper we shall consider the equilibrium configuration of these material particles where the pairwise potential is the more realistic Lennard-Jones potential and where the nearest neighbor interaction is not assumed in our discussion. We shall estimate the error of the QC approximation.

Consider a one-dimensional crystal material where $N$ atoms are distributed on a straight line. Let $u_{i}, i=0,1, \ldots, N$, denote the position of the $i$ th atom, and let $W_{i j}\left(u_{i j}\right)$ denote the embedding and interacting energy of atoms $i$ and $j$, where $u_{i j}=\left|u_{i}-u_{j}\right|$. We assume that the energy functions $W_{i j}$ between any two atoms are all the same, and denote it by $W$. Following Frank and van der Merwe, we adopt the so-called Lennard-Jones potential [3]

$$
W(\alpha)=-\left(\frac{\sigma}{\alpha}\right)^{6}+\left(\frac{\sigma}{\alpha}\right)^{12}
$$

for the one-dimensional lattice. $\sigma>0$ is something like the lattice scale. Also see Figure 1 for what the function looks like. Note that for $\alpha$ near zero the graphs of the function $W$ and its derivatives are too high or too low to be shown in the figure. 
Obviously $\gamma=\sqrt[6]{2} \sigma$ is the minimum point of the function $W(\alpha)$. We thus can write down the total potential energy of the material: $\frac{1}{2} \sum_{i=0}^{N} \sum_{j=0, j \neq i}^{N} W\left(u_{i j}\right)$. Stable configurations of the crystal material are identified by the minimizers of the potential energy subject to a stress-free boundary condition (i.e., the force acting on the boundary atoms is zero). For the sake of determination, we fix the left-end atom, say $u_{0}=0$, and let $u=\left(u_{1}, \ldots, u_{N}\right)^{T}$. Without loss of generality we let $u_{i}>u_{i-1}, i=1, \ldots, N$. So the problem in the full lattice scale is: find $\hat{u}$ such that

$$
E(\hat{u})=\min _{u} E(u)=\min _{u} \frac{1}{2} \sum_{i=0}^{N} \sum_{j=0, j \neq i}^{N} W\left(\left|u_{j}-u_{i}\right|\right)=\min _{u} \sum_{i=0}^{N} \sum_{j=i+1}^{N} W\left(u_{j}-u_{i}\right) .
$$

If we make a variable transformation $r_{i}=u_{i}-u_{i-1}, i=1,2, \ldots, N$, then we can write (2) in terms of $r=\left(r_{1}, \ldots, r_{N}\right)^{T}$ :

$$
\bar{E}(\hat{r})=\min _{r} \bar{E}(r),
$$

where

$$
\bar{E}(r)=\sum_{i=1}^{N} W\left(r_{i}\right)+\sum_{i=1}^{N-1} W\left(r_{i}+r_{i+1}\right)+\cdots+W\left(\sum_{j=1}^{N} r_{j}\right)=\sum_{j=1}^{N} \sum_{i=1}^{N+1-j} W\left(\sum_{k=i}^{i+j-1} r_{k}\right) .
$$

Existence, uniqueness and conditioning of the problem will be discussed in $\S 2$ based on a solution estimate

$$
\frac{4}{5} \sigma<r_{i} \leq \gamma
$$

The proof of (5) is not trivial, but it is a key to obtaining all results throughout the paper. Also in $\S 2$ we find that the problem in terms of the distance $r$ has better conditioning than that in terms of the position $u$.

Because of the shape of the function $W(\alpha)$, the interaction of particles is weaker as their distance becomes larger. Hence the potential energy generated by one particle, say the $i$ th particle, may count only the interaction with its nearby particles within a distance $\rho_{c}(\delta)$, where $\delta=\left|W\left(\rho_{c}\right)\right|$ is small and $\rho_{c}(\delta)$ is called a cut-off radius. $\left(u_{i}-\rho_{c}, u_{i}+\rho_{c}\right)$ is called a cut-off interval. Apparently we should let $\rho_{c}>\gamma$, since $W(\alpha)$ is not that small for $\alpha \leq \gamma$. The original full interaction model is largely simplified by this interaction cut-off. To analyze the error we introduce a cut-off fraction

$$
F_{c}=\frac{\rho_{c}(\delta)}{\sigma}>1
$$

Obviously, as $F_{c}$ grows the cut-off radius grows, and the simplified system approaches the original system. In $\S 3$ we shall discuss the properties of the simplified problem and prove the error estimates

$$
\left\|r-r^{c}\right\| \leq K F_{c}^{-5} \sigma \quad \text { or } \quad\left\|u-u^{c}\right\| \leq K(N \sigma) F_{c}^{-5},
$$

where $K$ is a generic constant and $r$ (or $u$ ) and $r^{c}$ (or $u^{c}$ ) are the solution of the original problem and the simplified problem, respectively. $\|\cdot\|$ denotes $\|\cdot\|_{\infty}$ or $\frac{1}{\sqrt{N}}\|\cdot\|_{2}$, and $N \sigma=O(1)$ if the material has finite length.

In $\S 4$ we apply the idea of quasicontinuum (QC) approximation combined with the finite element method to the simplified problem. The finite element setting is a little different from the usual one. The independent variable is the index set of the 
particles $i=\{0, \ldots, N\}$. For this one-dimensional crystal material we only need a local QC model which assumes that each element contains a whole cut-off interval (cf. 7]). That is, the number of particles in one element is $l>2 i_{c}(\delta)$, where $i_{c}(\delta)$ is the maximal number of particles in a half of a cut-off interval. We shall see that the analysis is already fairly complicated for the local QC approximation. Through the problem in terms of the distance $r^{c}$ and using a linear shape function for the finite element method we shall prove that for $\left[\frac{l}{2}\right] \geq 2 i_{c}$ (where $\left[\frac{l}{2}\right]$ stands for the integer part of the number $\frac{l}{2}$ ),

$$
\left\|R_{i_{k}}-R_{i_{k}}^{c}\right\| \leq K(N \sigma) \max \left\{\frac{\eta}{N}, \frac{F_{c}^{-6}}{m}\right\}, \quad k=1,2, \ldots, m,
$$

and

$$
\left\|U_{i_{k}}-u_{i_{k}}^{c}\right\| \leq K(N \sigma) \max \left\{\frac{\eta}{4} F_{c}^{-1}, F_{c}^{-6}\right\}, \quad k=1,2, \ldots, m
$$

where $\|\cdot\|$ is either $\|\cdot\|_{\infty}$ or $\frac{1}{\sqrt{m}}\|\cdot\|_{2}, \eta=2 W^{\prime}\left(\frac{8}{5} \sigma\right) \sigma / 15 \approx 0.027, m$ is the number of elements, $R_{i_{k}}$ (or $U_{i_{k}}$ ) is the solution of the QC approximation at the $k$ th element, $u_{i_{k}}^{c}$ is the $i_{k}$ th component of the solution of the simplified problem and $R_{i_{k}}^{c}=u_{i_{k}}^{c}-u_{i_{k-1}}^{c}$. Then the error between the local QC solution and the full-lattice solution can be immediately obtained by combining (7)-(9), i.e.,

$$
\left\|U_{i_{k}}-u_{i_{k}}\right\| \leq K(N \sigma)\left(\frac{\eta}{4} F_{c}^{-1}+F_{c}^{-5}\right), \quad k=1,2, \ldots, m
$$

Here we write the error estimate in terms of $F_{c}$ in order to avoid complicated notation. More precise results and their proofs will be given in $\S 4$. Again $N \sigma=$ $O(1)$ if the material has a finite length. More precise results (including those for $\left.\left[\frac{l}{2}\right] \leq 2 i_{c}\right)$ and their proofs will be given in $\S 4$ (see (50)-(52)) .

\section{AnAlysis of the MODEL PROBlem AND its SOlution}

We analyze the one-dimensional crystal material model (2) or (3). If we only consider nearest neighbor interaction, then the problem is trivial. In this case $\bar{E}(r)=\sum_{i=1}^{N} W\left(r_{i}\right)$. The minimum is $r_{i}=\gamma$, where $\gamma$ is the minimum point of the function $W(\alpha)$. We shall study the problem without the nearest-neighborinteraction assumption. There are several fundamental problems to be considered. They are boundedness, uniqueness and existence of the solution of problem (2) or (3). The existence of the solution is obvious, since the function $W$, and then $E$ or $\bar{E}$, is bounded below.

Theorem 1. At a (local or global) minimum or (local or global) maximum of $E(u)=\bar{E}(r), r_{i}=u_{i}-u_{i-1} \leq \gamma$ for any $i=1,2, \ldots, N$, where $\gamma=2^{\frac{1}{6}} \sigma \approx 1.12 \sigma$.

Proof. Suppose that we have two adjacent atoms such that $\left|u_{i_{0}}-u_{i_{0}-1}\right|>\gamma$. We can group all the atoms into two parts. Part I includes atoms $u_{i}, i=0,1, \ldots, i_{0}-1$ and part II includes atoms $u_{i}, i=i_{0}, \ldots, N$. It is easy to verify that $W(\alpha)$ increases if $\alpha>\gamma$. If we fix the relative distance of any two atoms in each group and shift all atoms in part II towards part I by a tiny distance, then the energy $E$ will decrease a bit, since the distance between atoms in part I and part II decreases and is larger than $\gamma$. Similarly, if we shift all atoms in part II away from part I by a tiny distance, the energy $E$ will increase a bit. So $E$ cannot reach the maximum or minimum at the current configuration. This proves the theorem. 


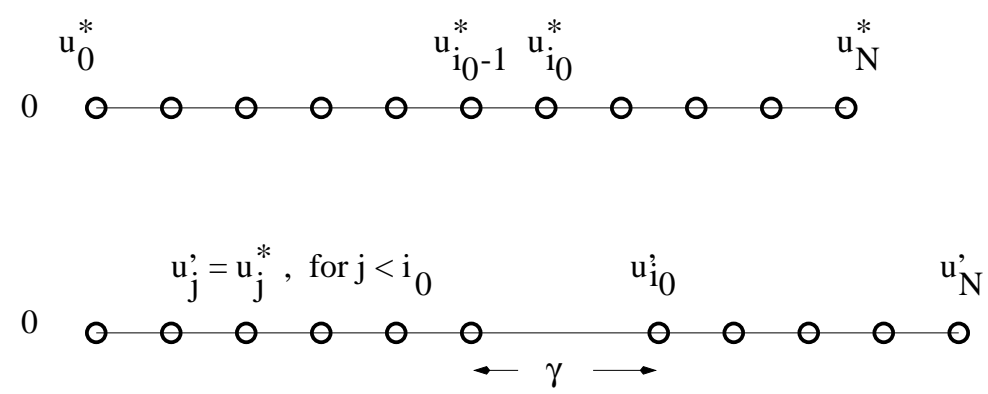

Figure 2. Configurations $u^{*}$ and $u^{\prime}$

From the result, we know that the solution of problem (21) or (3) is bounded by $N \gamma$ or the length of the material is of order $O(N \sigma)$. Next we give a lower bound for $r_{i}$.

Theorem 2. At the global minimum of the energy $E(u)=\bar{E}(r)$, the distance of any two adjacent particles should be away from zero, say, $r_{i}=u_{i}-u_{i-1}>\frac{4}{5} \sigma, i=$ $1,2, \ldots, N$.

Proof. We assume that at the minimum configuration $u^{*}=\left(u_{1}^{*}, \ldots, u_{N}^{*}\right)$ there exists an $i_{0}, 1 \leq i_{0} \leq N$, such that $u_{i_{0}}^{*}-u_{i_{0}-1}^{*} \leq \frac{4}{5} \sigma$ and, for all $i<i_{0}, u_{i}^{*}-$ $u_{i-1}^{*}>\frac{4}{5} \sigma$. We want to derive a contradiction out of the assumption, i.e., $E\left(u^{*}\right) \leq$ $E(u)$ for all $u$. The idea of the proof is to consider a specific configuration $u^{\prime}=$ $\left(u_{1}^{*}, \ldots, u_{i_{0}-1}^{*}, u_{i_{0}}^{\prime}, \ldots, u_{N}^{\prime}\right)$, where $u_{j}^{\prime}=u_{j}^{*}+\left[\gamma-\left(u_{i_{0}}^{*}-u_{i_{0}-1}^{*}\right)\right]$ for $j=i_{0}, \ldots, N$ (cf. Figure 2).

From $E\left(u^{*}\right) \leq E\left(u^{\prime}\right)$ we first show that there exists an interval of length $\gamma$ which includes 5 particles in it. In fact, if this is not true then every interval of length $\gamma$ includes at most 4 particles. Write down an equivalent total energy expression of (2):

$$
E\left(u^{\prime}\right)=\sum_{i=0}^{N} E_{i}\left(u^{\prime}\right), \text { where } E_{i}\left(u^{\prime}\right)=\sum_{j=i+1}^{N} W\left(u_{j}^{\prime}-u_{i}^{\prime}\right) .
$$

We scale the axis according to the length $\gamma$ starting from $u_{i_{0}}^{*}$ towards the right. Then in each length- $\gamma$ interval $\left[u_{i_{0}}^{*}+(k-1) \gamma, u_{i_{0}}^{*}+k \gamma\right], k=1,2, \ldots$, there are at most 4 atoms. We now compare $E\left(u^{\prime}\right)$ and $E\left(u^{*}\right)$ :

$$
\begin{aligned}
E_{0}\left(u^{\prime}\right) & =\sum_{j=1}^{i_{0}-1} W\left(u_{j}^{*}-u_{0}^{*}\right)+\sum_{j=i_{0}}^{N} W\left(u_{j}^{\prime}-u_{0}^{*}\right) \\
& =E_{0}\left(u^{*}\right)-\sum_{j=i_{0}}^{N} W\left(u_{j}^{*}-u_{0}^{*}\right)+\sum_{j=i_{0}}^{N} W\left(u_{j}^{\prime}-u_{0}^{*}\right) .
\end{aligned}
$$

Obviously $u_{j}^{\prime}-u_{0}^{*} \geq \gamma$ for $j \geq i_{0}$, since $u_{i_{0}}^{\prime}-u_{i_{0}-1}^{*}=\gamma$. So $\sum_{j=i_{0}}^{N} W\left(u_{j}^{\prime}-u_{0}^{*}\right)<0$. For the other sum we have

$$
-\sum_{j=i_{0}}^{N} W\left(u_{j}^{*}-u_{0}^{*}\right) \leq-4 W\left(\left(i_{0}-1\right) \frac{4}{5} \sigma\right)-4 \sum_{j=1}^{\infty} W\left(\left(i_{0}-1\right) \frac{4}{5} \sigma+j \gamma\right) .
$$


Note that if $i_{0}=1$ there is no $-4 W\left(\left(i_{0}-1\right) \frac{4}{5} \sigma\right)$ in above inequality. This gives

$$
E_{0}\left(u^{\prime}\right) \leq E_{0}\left(u^{*}\right)-4 W\left(\left(i_{0}-1\right) \frac{4}{5} \sigma\right)-4 \sum_{j=1}^{\infty} W\left(\left(i_{0}-1\right) \frac{4}{5} \sigma+j \gamma\right)
$$

Similarly, for $l=1, \ldots, i_{0}-3$, we have

$$
\begin{aligned}
E_{l}\left(u^{\prime}\right) & =E_{l}\left(u^{*}\right)-\sum_{j=i_{0}}^{N} W\left(u_{j}^{*}-u_{l}^{*}\right)+\sum_{j=i_{0}}^{N} W\left(u_{j}^{\prime}-u_{l}^{*}\right) \\
& \leq E_{l}\left(u^{*}\right)-4 W\left(\left(i_{0}-l-1\right) \frac{4}{5} \sigma\right)-4 \sum_{j=1}^{\infty} W\left(\left(i_{0}-l-1\right) \frac{4}{5} \sigma+j \gamma\right) .
\end{aligned}
$$

For $l=i_{0}-2$ and $l=i_{0}-1$,

$$
\begin{aligned}
& E_{i_{0}-2}\left(u^{\prime}\right) \leq E_{i_{0}-2}\left(u^{*}\right)-4 W(\gamma)-4 \sum_{j=1}^{\infty} W\left(\frac{4}{5} \sigma+j \gamma\right) \\
& E_{i_{0}-1}\left(u^{\prime}\right) \leq E_{i_{0}-1}\left(u^{*}\right)-W\left(u_{i_{0}}^{*}-u_{i_{0}-1}^{*}\right)-4 W(\gamma)-4 \sum_{j=1}^{\infty} W(j \gamma) .
\end{aligned}
$$

From $r_{i_{0}} \leq \frac{4}{5} \sigma$ we have $-W\left(u_{i_{0}}^{*}-u_{i_{0}-1}^{*}\right) \leq-W\left(\frac{4}{5} \sigma\right) \approx-10.74$. Also, by the construction of $u^{\prime}, E_{j}\left(u^{\prime}\right)=E_{j}\left(u^{*}\right)$ for all $j \geq i_{0}$. We thus obtain

$$
\begin{gathered}
E\left(u^{\prime}\right) \leq E\left(u^{*}\right)-10.74-2 \cdot 4 W(\gamma)-4 \sum_{l=0}^{i_{0}-3} W\left(\left(i_{0}-l-1\right) \frac{4}{5} \sigma\right) \\
-4 \sum_{l=0}^{i_{0}-1} \sum_{j=1}^{\infty} W\left(\left(i_{0}-l-1\right) \frac{4}{5} \sigma+j \gamma\right) \\
\leq E\left(u^{*}\right)-10.74-8 W(\gamma)-8 \sum_{l^{\prime}=1}^{\left[\left(i_{0}-3\right) / 2\right]} W\left(l^{\prime} \gamma\right) \\
-8 \sum_{l^{\prime}=0}^{\left[\left(i_{0}-1\right) / 2\right]} \sum_{j=1}^{\infty} W\left(l^{\prime} \gamma+j \gamma\right),
\end{gathered}
$$

where we have used the facts that $-W\left(\left(i_{0}-l-1\right) \frac{4}{5} \sigma\right) \leq-W\left(\frac{i_{0}-l-1}{2} \gamma\right)$ for $l=$ $0, \ldots, i_{0}-3$ and $-W\left(\left(i_{0}-l-1\right) \frac{4}{5} \sigma+j \gamma\right) \leq-W\left(\frac{i_{0}-l-1}{2} \gamma+j \gamma\right)$ for $l=0, \ldots, i_{0}-1$ and $j \geq 1$. We also have

$$
-\sum_{j=1}^{\infty} W(j \gamma)=-\sum_{j=1}^{3} W(j \gamma)-\sum_{j=4}^{\infty} W(j \gamma) \leq 0.2585+\frac{1}{2} \int_{3}^{\infty} \frac{1}{j^{6}} d j \approx 0.2593
$$


where we have used $\left(\frac{\sigma}{\gamma}\right)^{6}=\frac{1}{2}$ and $1-\left(\frac{\sigma}{j \gamma}\right)^{6} \leq 1$;

$$
\begin{aligned}
& -\sum_{j=1}^{\infty} W(\gamma+j \gamma)=-\sum_{j=1}^{\infty} W(j \gamma)+W(\gamma) \leq 0.0093 \\
& -\sum_{j=1}^{\infty} W(2 \gamma+j \gamma)=-\sum_{j=2}^{\infty} W(j \gamma)+W(2 \gamma) \leq 0.0016 \\
& -\sum_{l^{\prime}=3}^{\infty} \sum_{j=1}^{\infty} W\left(l^{\prime} \gamma+j \gamma\right) \leq \frac{1}{2} \sum_{l^{\prime}=3}^{\infty} \sum_{j=l^{\prime}+1}^{\infty} \frac{1}{j^{6}} \leq \frac{1}{2} \int_{2}^{\infty} \int_{j=l^{\prime}+1}^{\infty} \frac{1}{j^{6}} d j d l^{\prime} \approx 0.0016
\end{aligned}
$$

Hence,

$$
\begin{aligned}
E\left(u^{\prime}\right) & \leq E\left(u^{*}\right)-10.74+8 \cdot 0.2593+8 \cdot 0.25+8 \cdot 0.2718 \\
& =E\left(u^{*}\right)-4.4912<E\left(u^{*}\right) .
\end{aligned}
$$

This shows that $E\left(u^{*}\right)$ is not the minimum. This contradiction implies that there exists at least one interval of length $\gamma$ which includes 5 particles. Then there must exist two adjacent particles, say, $u_{i_{1}-1}^{*}, u_{i_{1}}^{*}$, such that $u_{i_{1}}^{*}-u_{i_{1}-1}^{*} \leq \frac{\gamma}{4}$. Therefore, we can assume that there exists an $i_{1}$ such that

$$
u_{i_{1}}^{*}-u_{i_{1}-1}^{*} \leq \frac{\gamma}{4}, \text { but } u_{i}^{*}-u_{i-1}^{*}>\frac{\gamma}{4} \text { for all } i<i_{1} .
$$

Note that $W\left(u_{i_{1}}^{*}-u_{i_{1}-1}^{*}\right) \geq W\left(\frac{1}{4} \gamma\right)=4^{11}\left(1-\left(\frac{4}{\sqrt[6]{2}}\right)^{-6}\right) \approx 4^{11}$.

Similarly to the previous argument, and from $E\left(u^{*}\right) \leq E\left(u^{\prime}\right)$, we can obtain (ignoring many algebraic operations) that there exists an interval of length $\gamma$ including $4^{2}+1$ particles. In other words, there must exist two adjacent particles, say, $u_{i_{2}-1}^{*}$, $u_{i_{2}}^{*}$, such that $u_{i_{2}}^{*}-u_{i_{2}-1}^{*} \leq \frac{1}{4^{2}} \gamma$. Repeating this argument, we conclude that there exists an interval of length $\gamma$ which includes as many particles as you want if $E\left(u^{*}\right)$ is the minimum. This contradicts the fact that the number of particles or atoms is finite. This completes the proof of the theorem.

Theorems 1 and 2 show that (5) holds. From (5) we can obtain the uniqueness of the solution of (2) or (3).

Theorem 3. The (local) maximum and (local) minimum of the energy $\bar{E}(r)=$ $E(u)$ is unique in the region $\mathbf{R}: \frac{4}{5} \sigma \leq r_{i} \leq \gamma, i=1,2, \ldots, N$.

Proof. At the maximum or minimum of $\bar{E}(r)$ (cf. (4)), $r$ should satisfy

$$
\begin{aligned}
\frac{\partial \bar{E}}{\partial r_{i}}=W^{\prime}\left(r_{i}\right) & +\sum_{j=(i-1) \vee 1}^{i \wedge(N-1)} W^{\prime}\left(r_{j}+r_{j+1}\right) \\
& +\sum_{j=(i-2) \vee 1}^{i \wedge(N-2)} W^{\prime}\left(\sum_{k=0}^{2} r_{j+k}\right)+\cdots+W^{\prime}\left(\sum_{j=1}^{N} r_{j}\right)=0,
\end{aligned}
$$

where $a \vee b$ and $a \wedge b$ represent $\max (a, b)$ and $\min (a, b)$, respectively.

If the Hessian matrix of $\bar{E}(r)$ is diagonally dominant in the region $\mathbf{R}$, then the matrix is nonsingular in the region. We thus prove that the system (13) has a unique solution in the region (i.e., the minimum of $\bar{E}(r)$ is unique). So we only 
need to show that the Hessian matrix of $\bar{E}(r)$ is diagonally dominant in the region R. The diagonal element of the Hessian matrix of $\bar{E}(r)$ is

$$
\begin{aligned}
\frac{\partial^{2} \bar{E}}{\partial r_{i}^{2}}=W^{\prime \prime}\left(r_{i}\right) & +\sum_{j=(i-1) \vee 1}^{i \wedge(N-1)} W^{\prime \prime}\left(r_{j}+r_{j+1}\right) \\
& +\sum_{j=(i-2) \vee 1}^{i \wedge(N-2)} W^{\prime \prime}\left(\sum_{k=0}^{2} r_{j+k}\right)+\cdots+W^{\prime \prime}\left(\sum_{j=1}^{N} r_{j}\right) .
\end{aligned}
$$

In the region $\mathbf{R}, r_{i} \leq \gamma$. Hence, $W^{\prime \prime}\left(r_{i}\right) \geq W^{\prime \prime}(\gamma) \approx 15 / \sigma^{2}$, since $W^{\prime \prime}(\alpha)$ decreases when $\alpha \leq \gamma$. We also have

$$
r_{j}+r_{j+1}>2\left(\frac{4}{5} \sigma\right)>\gamma, r_{j}+r_{j+1}+r_{j+2}>3\left(\frac{4}{5} \sigma\right), \ldots
$$

Hence, from the monotone property of $W^{\prime \prime}(\alpha)$, all other terms in the right hand side of (14) except $W^{\prime \prime}\left(r_{i}\right)$ are negative and the sum of them is larger than $A=$ $2 W^{\prime \prime}\left(\frac{8}{5} \sigma\right)+3 W^{\prime \prime}\left(\frac{12}{5} \sigma\right)+\cdots$. Noting that $W^{\prime \prime}\left(\frac{8}{5} \sigma\right) \approx-0.7614 / \sigma^{2}, W^{\prime \prime}\left(\frac{12}{5} \sigma\right) \approx$ $-0.0375 / \sigma^{2}, W^{\prime \prime}\left(\frac{16}{5} \sigma\right) \approx-0.0038 / \sigma^{2}, \ldots$, we have

$$
\begin{aligned}
0 \geq A & =\sum_{j=2}^{4} j W^{\prime \prime}\left(j \frac{4}{5} \sigma\right)+\sum_{j=5}^{N} j W^{\prime \prime}\left(j \frac{4}{5} \sigma\right) \\
& >-1.6505 / \sigma^{2}-7\left(\frac{5}{4}\right)^{8} \int_{4}^{\infty} \frac{6}{x^{7}} d x / \sigma^{2} \geq-1.7 / \sigma^{2} .
\end{aligned}
$$

At the same time we can calculate off-diagonal elements $\frac{\partial^{2} \bar{E}}{\partial r_{i} \partial r_{j}}(j \neq i)$, and all these off diagonal elements can be estimated using (15). We then obtain that the sum of the absolute values of all off-diagonal elements of the Hessian matrix of $\bar{E}(r)$ is less than

$$
\begin{aligned}
B & \leq-2 W^{\prime \prime}\left(2 \cdot \frac{4}{5} \sigma\right)-6 W^{\prime \prime}\left(3 \cdot \frac{4}{5} \sigma\right)-12 W^{\prime \prime}\left(4 \cdot \frac{4}{5} \sigma\right)-\sum_{j=5}^{\infty} j(j-1) W^{\prime \prime}\left(j \cdot \frac{4}{5} \sigma\right) \\
& \leq 1.7934 / \sigma^{2}+7\left(\frac{5}{4}\right)^{8} \int_{4}^{\infty} \frac{x-1}{x^{7}} d x / \sigma^{2} \leq 1.8 / \sigma^{2} .
\end{aligned}
$$

So the diagonal element is larger than $15 / \sigma^{2}-|A| \geq 13.7 / \sigma^{2}>B$. Therefore, each row of the Hessian matrix of $\bar{E}(r)$ is diagonally dominant in the region $\mathbf{R}$. This completes the proof.

Since the global minimum of $\bar{E}(r)=E(u)$ exists, the theorems above imply that the global minimum of $\bar{E}(r)$ is located in the region $\mathbf{R}$ and the last theorem implies that $\bar{E}(r)$ has no other critical values in $\mathbf{R}$. Hence, the global minimum is unique in $\mathbf{R}$, since a global minimum can be a local minimum of a slightly larger region and Theorem 3 holds in the slightly larger region as well.

Remark 1. The second derivative (Hessian matrix) $\nabla_{r}^{2} \bar{E}(r)$ of $\bar{E}(r)$ with respect to $r$ is symmetric. In the above theorem we have shown that $\nabla_{r}^{2} \bar{E}(r)$ is diagonally dominant and the diagonal elements are all positive in the region $\mathbf{R}$. The eigenvalues of the Hessian matrix are thus all positive by Gershgorin's theorem. This implies that $\nabla_{r}^{2} \bar{E}(r)$ is positive definite in the region $\mathbf{R}$. Using Gershgorin's theorem and 
the off-diagonal sum we calculated earlier, we can actually have a lower bound of the eigenvalues (say, $\lambda_{i}, i=1, \ldots, N$ ) of the Hessian matrix, i.e.,

$$
\lambda_{i} \geq(15-1.7-1.8) / \sigma^{2}=11.5 / \sigma^{2} .
$$

Similarly we can also have $\lambda_{i} \leq(15+1.7+1.8) / \sigma^{2}=17.5 / \sigma^{2}$. So problem (3) is well-posed.

Corollary 1. E(u) has a unique minimum, and in the region $\mathbf{R}$ its second derivative is positive definite too. Hence, it has no any other critical values in $\mathbf{R}$.

Proof. We have $r_{i}=u_{i}-u_{i-1}, i=1, \ldots, N$. So $r=T u$, where

$$
T=\left(\begin{array}{ccccc}
1 & & & & \\
-1 & 1 & & & \\
& \ldots & \ldots & & \\
& & -1 & 1 & \\
& & & -1 & 1
\end{array}\right) .
$$

It is not difficult to verify that

$$
\nabla_{u}^{2} E(u)=T^{T} \nabla_{r}^{2} E(r) T .
$$

So in the region $\mathbf{R}, \nabla_{u}^{2} E(u)$ is positive definite since $\nabla_{r}^{2} E(r)$ is. This fact also shows that $E(u)$ has only one minimum in $\mathbf{R}$, and there are no other critical values.

Theorem 4. The smallest and the largest eigenvalues of the matrix $\nabla_{u}^{2} E(u)$ are of order $O\left(1 /(N \sigma)^{2}\right.$ and $O\left(1 / \sigma^{2}\right)$, respectively.

Proof. The smallest and largest eigenvalues of a symmetric positive definite matrix $B$ can be expressed as

$$
\lambda_{\min }=\min _{x \neq 0} \frac{x^{T} B x}{x^{T} x}, \quad \lambda_{\max }=\max _{x \neq 0} \frac{x^{T} B x}{x^{T} x} .
$$

From (16),

$$
x^{T} \nabla_{u}^{2} E(u) x=x^{T} T^{T} \nabla_{r}^{2} E(r) T x .
$$

Using Remark 1, we thus have

(18) $x^{T} \nabla_{u}^{2} E(u) x=(T x)^{T} \nabla_{r}^{2} E(r)(T x)=O\left(\frac{1}{\sigma^{2}}\right)(T x)^{T}(T x)=O\left(\frac{1}{\sigma^{2}}\right) x^{T} T^{T} T x$,

where

$$
x^{T} T^{T} T x=x_{1}^{2}+\sum_{i=1}^{N-1}\left(x_{i+1}-x_{i}\right)^{2} \leq 4 x^{T} x .
$$

We can choose a special $x_{s}=(1,0, \ldots, 0)^{T}$ such that $x_{s}^{T} T^{T} T x_{s}=2 x_{s}^{T} x_{s}$. So from (17) the largest eigenvalue of $\nabla_{u}^{2} E(u)$ is of order $O\left(1 / \sigma^{2}\right)$.

Now we consider the smallest eigenvalue. Assume $x_{i_{0}}=\max _{i}\left|x_{i}\right|$. Hence, $x^{T} x \leq$ $N x_{i_{0}}^{2}$. Let $y_{i}=\frac{x_{i}}{x_{i_{0}}}, i=1,2, \ldots, N$. We have (noting that $\left|y_{i}\right| \leq 1$ )

$$
\begin{aligned}
& \frac{x^{T} T^{T} T x}{x^{T} x} \geq \frac{1}{N}\left(\left(y_{N}-y_{N-1}\right)^{2}+\cdots+\left(y_{i_{0}+1}-1\right)^{2}\right. \\
& \left.\quad+\left(1-y_{i_{0}-1}\right)^{2}+\cdots+\left(y_{2}-y_{1}\right)^{2}+y_{1}^{2}\right) \geq \frac{1}{N i_{0}} .
\end{aligned}
$$


Hence, $x^{T} T^{T} T x \geq \frac{1}{N^{2}} x^{T} x$. Also, we can choose a vector $x_{p}=(1,2, \ldots, N)^{T}$ such that

$$
\frac{x_{p}^{T} T^{T} T x_{p}}{x_{p}^{T} x_{p}}=\frac{6}{(N+1)(2 N+1)}=O\left(1 / N^{2}\right) .
$$

From (17) and (18) we obtain that the smallest eigenvalue of $\nabla_{u}^{2} E(u)$ is of order $O\left(1 /(N \sigma)^{2}\right)$.

Remark 2. The above theorem implies that problem (2) is ill-posed when $N$ is large, since the condition number of its Hessian matrix $\frac{\lambda_{\max }}{\lambda_{\min }}$ is $O\left(N^{2}\right)$.

\section{Cut-OFF-A SimplificAtion OF THE MODEL}

By the shape of the potential $W(\alpha)$, it may be good enough to consider only the interaction of one particle with its nearby particles within the cut-off radius $\rho_{c}$. In this section we shall prove error estimates (7) of such simplification in terms of the cut-off fraction $F_{c}$ (see (6) ). We have shown that $\frac{4}{5} \sigma<r_{i} \leq \gamma=\sqrt[6]{2} \sigma$. We can then obtain

$$
\frac{1}{\sqrt[6]{2}} F_{c} \leq i_{c}(\delta) \leq \frac{5}{4} F_{c}
$$

We also roughly have

$$
\left|W\left(i_{c} \frac{4}{5} \sigma\right)\right| \approx \delta \approx F_{c}^{-6} .
$$

The total energy after the cut-off is

$$
E_{c}\left(u^{c}\right)=\frac{1}{2} \sum_{i=0}^{N} \sum_{j=i-i_{c}(\delta) j \neq i}^{i+i_{c}(\delta)} W\left(\left|u_{j}^{c}-u_{i}^{c}\right|\right),
$$

or, in terms of $r^{c}$,

$$
\begin{aligned}
\bar{E}_{c}\left(r^{c}\right)= & \sum_{i=1}^{N} W\left(r_{i}^{c}\right)+\sum_{i=1}^{N-1} W\left(r_{i}^{c}+r_{i+1}^{c}\right) \\
& +\cdots+\sum_{i=1}^{N-i_{c}+1} W\left(r_{i}^{c}+r_{i+1}^{c}+\cdots+r_{i+i_{c}-1}^{c}\right) \\
= & \sum_{j=1}^{i_{c}} \sum_{i=1}^{N+1-j} W\left(\sum_{k=i}^{i+j-1} r_{k}\right),
\end{aligned}
$$

where $u^{c}=\left(u_{1}^{c}, u_{2}^{c}, \ldots, u_{N}^{c}\right)^{T}, u_{0}^{c}=0$ and $r^{c}=\left(r_{1}^{c}, \ldots, r_{N}^{c}\right)^{T}, r_{i}^{c}=u_{i}^{c}-u_{i-1}^{c}$. Hence the simplified problem of (2) or (3) is finding $\hat{r}^{c}$ such that

$$
\bar{E}_{c}\left(\hat{r}^{c}\right)=\min _{r^{c}} \bar{E}_{c}\left(r^{c}\right) .
$$

(In terms of $u^{c}$, the problem is $E_{c}\left(\hat{u}^{c}\right)=\min _{u^{c}} E_{c}\left(u^{c}\right)$.)

Problem (23) is not same as the original problem (3). Obviously, Theorem 1 is no longer true for all local minima or maxima. The distance between two adjacent particles may be larger than $\gamma$ at some local minimum or maximum. For example, we could have $r_{i}^{c}>\rho_{c}$ for some $i$, and the system is divided into two independent subsystems at this $i$. There is no interaction between these two subsystems. So problem (23) could have many local minima which are not the minimum of the original system since $r_{i}^{c} \leq \gamma$ is not satisfied. However, we will prove that there exists 
a unique solution of problem (23) in the region $\mathbf{R}_{c}$ where the distance between any two adjacent particles is strictly less than the cut-off radius $\rho_{c}$, i.e.,

$$
\mathbf{R}_{c}=\left\{r^{c}: r_{i}^{c}<\rho_{c}, i=1,2, \ldots, N\right\} .
$$

Once we have the existence of the minimum solution in the region $\mathbf{R}_{c}$, then it should be the global minimum of the problem (23) for any $r^{c}$, since the minimum of the subsystem is larger than the minimum of the whole system.

Theorem 5. If $r^{c}$ or $u^{c}$ is in $\mathbf{R}_{c}$ then, at the minimum of the energy $\bar{E}_{c}\left(r^{c}\right)$ or $E_{c}\left(u^{c}\right)$, the distance between any two adjacent particles should satisfy $\gamma>r_{i}^{c}=$ $u_{i}^{c}-u_{i-1}^{c}>\frac{4}{5} \sigma$ for $i=1,2, \ldots, N$.

Proof. The proof is just the same as that for Theorems 1 and 2, except for restricting $u^{c}$ in $\mathbf{R}_{c}$ in proving the first part of the inequality.

Theorem 6. Problem (23) has a unique solution for $r^{c} \in \mathbf{R}_{c}$.

Proof. The existence of the global minimum of (23) is obvious, since each term in the expression of $\bar{E}_{c}$ has a lower bound. Following the idea of the proof in Theorem 1, the global minimum must be in $\mathbf{R}_{c}$. From the previous theorem we thus have $r^{c} \in \mathbf{R}$. Following the proof of Theorem 3 step by step, we can obtain that the Hessian matrix is symmetric positive definite in the region $\mathbf{R}$. Hence, the solution is unique.

From Theorem 4 of the previous section the condition number of problem (2) is worse than that of problem (3), although they are mathematically equivalent. So it is better to solve problem (3). Next we prove the error estimate (7) for $r-r^{c}$, and then give the corresponding results (7) for $u-u^{c}$ using the relation $r-r^{c}=T\left(u-u^{c}\right)$.

Theorem 7. Let $r^{c}$ be the solution of (23) and $r$ the solution of (3). We have the first estimate of (7).

Proof. The solution of (23) satisfies

$$
\frac{\partial \bar{E}_{c}}{\partial r_{i}^{c}}=W^{\prime}\left(r_{i}^{c}\right)+\sum_{j=(i-1) \vee 1}^{i \wedge(N-1)} W^{\prime}\left(r_{j}^{c}+r_{j+1}^{c}\right)+\cdots+\sum_{j=\left(i-i_{c}\right) \vee 1}^{i \wedge\left(N-i_{c}\right)} W^{\prime}\left(\sum_{k=0}^{i_{c}-1} r_{j+k}^{c}\right)=0
$$

for $i=1,2, \ldots, N$. The solution of (3) satisfies (13). Subtracting (25) from (13) and applying Taylor's theorem, we have

$$
H_{c}(\psi)\left(r-r^{c}\right)=g
$$

where $\psi=\theta r+(1-\theta) r^{c}, 0<\theta<1, H_{c}(\psi)=\nabla_{r^{c}}^{2} \bar{E}_{c}(\psi)$ (cf. (14)) and $g=$ $\left(g_{1}, \ldots, g_{N}\right)^{T}$, where

$$
\left|g_{i}\right|=\sum_{j=\left(i-i_{c}-1\right) \vee 1}^{i \wedge\left(N-i_{c}-1\right)} W^{\prime}\left(r_{j}+\cdots+r_{j+i_{c}}\right)+\cdots+W^{\prime}\left(r_{1}+\cdots+r_{N}\right) .
$$

Note that $r$ and $r^{c}$ both belong to the region $\mathbf{R}$. So $\psi \in \mathbf{R}$. Similarly to the proof of Theorem 3, we can have that $H_{c}(\psi)$ is strictly diagonally dominant and its diagonal elements $\left(\right.$ say, $d_{i}$ ) are larger than $11.5 / \sigma^{2}$. Write $H_{c}=D+F=D\left(I+D^{-1} F\right)$, 
where $D=\left(d_{i}\right)$ is the diagonal part of $H_{c}$ and $F$ is the off-diagonal part of $H_{c}$. Strictly diagonal dominance of $H_{c}$ gives $\left\|D^{-1} F\right\|_{\infty}<1$. Therefore

$$
\left\|H_{c}^{-1}\right\|_{\infty}=\left\|\left(I+D^{-1} F\right)^{-1} D^{-1}\right\|_{\infty} \leq \frac{\left\|D^{-1}\right\|_{\infty}}{1-\left\|D^{-1} F\right\|_{\infty}} \leq K \sigma^{2} .
$$

Now we estimate $g$ :

$$
\begin{aligned}
\left|g_{i}\right| & \leq\left(i_{c}+1\right) W^{\prime}\left(\left(i_{c}+1\right) \frac{4}{5} \sigma\right)+\left(i_{c}+2\right) W^{\prime}\left(\left(i_{c}+2\right) \frac{4}{5} \sigma\right)+\cdots \\
& =\sum_{j=i_{c}+1}^{\infty} j W^{\prime}\left(j \frac{4}{5} \sigma\right) \\
& \leq \frac{1}{\sigma}\left(\frac{4}{5}\right)^{7} \int_{i_{c}}^{\infty} \frac{6}{j^{6}} d j=\frac{6}{5}\left(\frac{4}{5}\right)^{7} \frac{1}{\sigma} \frac{1}{i_{c}^{5}} \\
& \leq \frac{48}{25} i_{c}\left|W\left(i_{c} \frac{4}{5} \sigma\right)\right| \leq K F_{c}^{-5} / \sigma
\end{aligned}
$$

where we have used (19) and (20). This completes the proof of the theorem for the norm $\|\cdot\|_{\infty}$. The result for the norm $\frac{1}{N}\|\cdot\|_{2}$ follows immediately.

Remark 3. Since $r=T u$ and $r^{c}=T u^{c}$, we have $u-u^{c}=T^{-1}\left(r-r^{c}\right)$. From the proof of Theorem 4 we have $x^{T} T T^{T} x \geq \frac{1}{N^{2}} x^{T} x$. This implies that $\left\|T^{-1}\right\|_{2} \leq N$. Furthermore, we can actually find $T^{-1}$, which is an lower triangular matrix whose lower triangular elements are all 1 . That means $\left\|T^{-1}\right\|_{\infty}=N$. Hence we have the second estimate of (17). Note that $N \sigma=O(1)$ if we assume the material is of finite length.

\section{The QUASICONTINUUM APPROXIMATION AND ITS ERROR ESTIMATES}

To further reduce the size of the problem we consider the local QC approximation combined with a finite element method for problem (23) in this section. For simplicity, we partition the 1-D material into $m$ parts (elements) such that there are $l$ particles in each part. So the total number of particles is $N=m l$. If the number of particles is not equal in each element, our argument can still be used without much difficulty. A middle particle of each element is a finite element node. We denote its position by $U_{i_{k}}, k=1, \ldots, m$. We assume that the deformation of particles between any two adjacent nodes (i.e., $\left.\left(U_{i_{k-1}}, U_{i_{k}}\right), k=2, \ldots, m\right)$ is homogeneous, that is, the distance between any two adjacent particles in an interval $\left(U_{i_{k-1}}, U_{i_{k}}\right)$ is the same. For the 1-D material we consider in this paper there are no inhomogeneous effects. Local QC approximation is thus suggested (cf. [7]); that is, we assume $l>2 i_{c}(\delta)$. We will see later from the error estimate that the local approximation is good enough for our problem.

A more precise description is the following. Applying the finite element idea to the index set $\{i=0, \ldots, N\}$ of a large number of points (or positions of particles), we write

$$
U_{i}=\sum_{k=1}^{m} U_{i_{k}} \phi_{k}(i)
$$



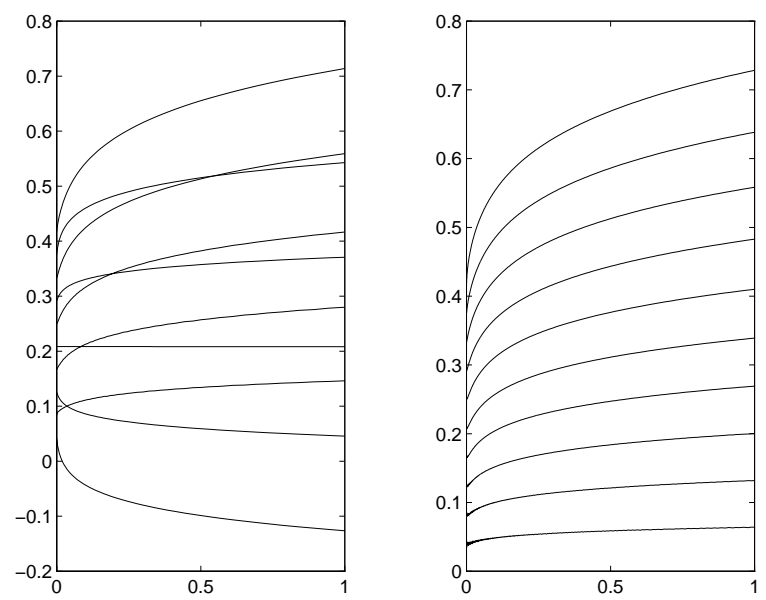

Figure 3 . The deformation gradient average and the energy average solutions of $\frac{d u}{d t}=\nabla E(u)$

where the $\phi_{k}$ are shape functions of $i$, and $U_{i_{k}}(k=1, \ldots, m)$ is the position of the $k$ th node particle in the $k$ th element. Now if we use linear shape functions, then

$$
\phi_{k}=\left\{\begin{array}{cc}
0 & \text { if } i \leq i_{k-1}, i \geq i_{k+1}, \\
\frac{i-i_{k-1}}{a_{k}} & \text { if } i_{k-1} \leq i \leq i_{k}, \\
\frac{i_{k+1}-i}{a_{k+1}} & \text { if } i_{k} \leq i \leq i_{k+1}
\end{array}\right.
$$

and

$$
U_{j}-U_{i_{k}}= \begin{cases}\frac{i_{k}-j}{a_{k}}\left(U_{i_{k}}-U_{i_{k-1}}\right) & \text { if } j<i_{k}, \\ \frac{j-i_{k}}{a_{k+1}}\left(U_{i_{k+1}}-U_{i_{k}}\right) & \text { if } j>i_{k},\end{cases}
$$

where $U_{j}$ is in the $k$ th element, and $a_{k}=i_{k}-i_{k-1}, k=1, \ldots, m+1$. Under our assumption we simply have $a_{k}=\left[\frac{l}{2}\right]$ for $k=1, m+1$ and $a_{k}=l$ for other $k$. If we make another approximation, namely that the potential energy generated by every particle in one element is equal, then we are able to write down the approximate total energy of the material. But in each element, say the $k$ th, the deformation gradient $S_{k}=\frac{U_{i_{k}}-U_{i_{k-1}}}{a_{k}}$ in $\left[i_{k}-\frac{a_{k}}{2}, i_{k}\right]$ is different from the deformation gradient $S_{k+1}=\frac{U_{i_{k+1}}-U_{i_{k}}}{a_{k+1}}$ in the other part $\left[i_{k}, i_{k}+\frac{a_{k+1}}{2}\right]$ of the element. To make the approximate solution smoother in each element, some kind of average is suggested in [7]. One way is to average two deformation gradients in each element, and calculate the potential of the $j$ th and the $i_{k}$ th particles as $W\left(\frac{S_{k}+S_{k+1}}{2}\left(j-i_{k}\right)\right)$. The other is to average the energies in two parts of each element using those two deformation gradients. The former should not be used, since it allows one particle to cross the other, which is not physical. Figure 3 is an example of ten particles to show that the average of deformation gradients allows such an unphysical crossing, while the other way does not. 
So we use the latter (i.e., the energy average form), and the approximate total energy can be written as

$$
\begin{aligned}
\tilde{E}^{r} & =l \sum_{k=1}^{m} \sum_{j=i_{k}-i_{c}(\sigma), j \neq i_{k}}^{i_{k}+i_{c}(\sigma)} W\left(\left|U_{j}-U_{i_{k}}\right|\right) \\
& =\frac{l}{2} \sum_{k=1}^{m}\left(\sum_{j=i_{k}-i_{c}}^{i_{k}-1} W\left(\frac{i_{k}-j}{a_{k}} R_{i_{k}}\right)+\sum_{j=i_{k}+1}^{i_{k}+i_{c}} W\left(\frac{j-i_{k}}{a_{k+1}} R_{i_{k+1}}\right)\right),
\end{aligned}
$$

where $R_{i_{k}}=U_{i_{k}}-U_{i_{k-1}}$ for $k=1,2, \ldots, m$, and we denote $R=\left(R_{i_{1}}, \ldots, R_{i_{m}}\right)^{T}$. For particles numbered in $\left[i_{m}, N\right]$ the deformation gradient is assumed to be the same as $S_{m}$ or $\frac{R_{i_{m+1}}}{a_{m+1}}=\frac{R_{i_{m}}}{a_{m}}$ for simplicity. The approximate problem is to find $\hat{R}$ such that

$$
\tilde{E}^{r}(\hat{R})=\min _{R} \tilde{E}^{r}(R)
$$

Similarly to the discussion for (23), problem (32) may have an unphysical solution as well if $U_{j}-U_{j-1}=R_{i_{k}} / a_{k}>\rho_{c}$., In this case the problem is broken into two subproblems for nodes $\left\{U_{i_{1}}, \ldots, U_{i_{k-1}}\right\}$ and $\left\{U_{i_{k}}, \ldots, U_{i_{m}}\right\}$. We thus can have one solution of (32) by solving the subproblems. But the solution is not what we want since $U_{j}-U_{j-1} \gg \gamma$ in the $k$ th element. As we did for (23), we consider a region

$$
\mathbf{R}_{q c}=\left\{R_{i_{k}}: R_{i_{k}} / a_{k}<\rho_{c}, k=1,2, \ldots, m .\right\} .
$$

If $\left\{R_{i_{k}}\right\}_{k=1}^{m} \in \mathbf{R}_{q c}$, then $\left\{R_{j}=U_{j}-U_{j-1}\right\}_{j=1}^{N} \in \mathbf{R}_{c}$. Using the technique in proving Theorems 1, 2 and 5, we can prove that the solution of (32) in $\mathbf{R}_{q c}$ satisfies

$$
\frac{4}{5} \sigma \leq U_{j}-U_{j-1}=R_{i_{k}} / a_{k} \leq \gamma \text { for all } k,
$$

where the integer $j \in\left(i_{k-1}, i_{k}\right]$. From the estimates of the solution we are able to prove the uniqueness and existence of the solution of (32) in the region $\mathbf{R}_{q c}$ similarly to previous arguments. However, we shall focus on error estimates.

The solution $R_{i_{k}}$ should satisfy

$$
\begin{aligned}
\frac{\partial \tilde{E}^{r}}{\partial R_{i_{k}}}= & \frac{1}{2}\left(\sum_{j=i_{k}-i_{c}}^{i_{k}-1} W^{\prime}\left(\frac{i_{k}-j}{a_{k}} R_{i_{k}}\right)\left(i_{k}-j\right)\right. \\
& \left.+\sum_{j=i_{k-1}+1}^{i_{k-1}+i_{c}} W^{\prime}\left(\frac{j-i_{k-1}}{a_{k}} R_{i_{k}}\right)\left(j-i_{k-1}\right)\right) \\
= & \sum_{j=i_{k}-i_{c}}^{i_{k}-1} W^{\prime}\left(\frac{i_{k}-j}{a_{k}} R_{i_{k}}\right)\left(i_{k}-j\right)=0
\end{aligned}
$$

for $k=2, \ldots, m-1$. We can easily verify that the last equality of (35) also holds for $k=1$ and $k=m$. So we have (35) for all $k=1, \ldots, m$. The total energy after the cut-off can be written as

$$
\bar{E}_{c}=\frac{1}{2} \sum_{i=0}^{N}\left(\sum_{j=i-i_{c} \vee 1}^{i-1} W\left(r_{i}^{c}+\cdots+r_{j+1}^{c}\right)+\sum_{j=i+1}^{i+i_{c} \wedge N} W\left(r_{j}^{c}+\cdots+r_{i+1}^{c}\right)\right),
$$

where we have used $u_{j}^{c}-u_{i}^{c}=r_{i}^{c}+\cdots+r_{j+1}^{c}$ for $j>i$ and $u_{i}^{c}-u_{j}^{c}=r_{j}^{c}+\cdots+r_{i+1}^{c}$ for $j<i$. 
We now compare the solution of minimization problem (32) to that of (36) or (23). From error estimates for $R_{i_{k}}$ we can then obtain those for $U_{i_{k}}$. The solution $r_{i}^{c}$ that minimizes $\bar{E}_{c}$ should satisfy

$$
\begin{aligned}
\frac{\partial \bar{E}_{c}}{\partial r_{i}^{c}}= & \frac{1}{2} \sum_{p=0}^{i_{c}-1} \sum_{j=i-i_{c}+p}^{i-1} W^{\prime}\left(r_{i+p}^{c}+\cdots+r_{j+1}^{c}\right) \\
& +\frac{1}{2} \sum_{p=1}^{i_{c}} \sum_{j=i}^{i+i_{c}-p} W^{\prime}\left(r_{j}^{c}+\cdots+r_{i-p+1}^{c}\right)=0 .
\end{aligned}
$$

Let $R_{i_{k}}^{c}=u_{i_{k}}^{c}-u_{i_{k-1}}^{c}$ and $\bar{r}_{i}^{c}=R_{i_{k}}^{c} / a_{k}$ for all $i \in\left(i_{k-1}, i_{k}\right)$. Take $i=i_{k}$ in (37) and do Taylor's expansion at $R_{i_{k}}^{c} / a_{k}$ for each component of $r^{c}$ involved. We then obtain

$$
\sum_{j=i_{k}-i_{c}}^{i_{k}-1}\left(i_{k}-j\right) W^{\prime}\left(\frac{i_{k}-j}{a_{k}} R_{i_{k}}^{c}\right)=h_{i_{k}}
$$

where

$$
\begin{aligned}
h_{i_{k}}= & \frac{1}{2} \sum_{p=0}^{i_{c}-1} \sum_{j=i_{k}-i_{c}+p}^{i_{k}-1} W^{\prime \prime}\left(\psi_{j}^{1}\right)\left(r_{i_{k}+p}^{c}+\cdots+r_{j+1}^{c}-\left(i_{k}+p-j\right) R_{i_{k}}^{c} / a_{k}\right) \\
& +\frac{1}{2} \sum_{p=1}^{i_{c}} \sum_{j=i_{k}}^{i_{k}+i_{c}-p} W^{\prime \prime}\left(\psi_{j}^{2}\right)\left(r_{j}^{c}+\cdots+r_{i_{k}-p+1}^{c}-\left(j-i_{k}+p\right) R_{i_{k}}^{c} / a_{k}\right) .
\end{aligned}
$$

Here

$$
\psi_{j}^{1}=\theta_{1}\left(r_{i+k}^{c}+\cdots+r_{j+1}^{c}\right)+\left(1-\theta_{1}\right)\left(i_{k}+p-j\right) R_{i_{k}}^{c} / a_{k}
$$

and

$$
\psi_{j}^{2}=\theta_{2}\left(r_{j}^{c}+\cdots+r_{i-k+1}^{c}\right)+\left(1-\theta_{2}\right)\left(\left(j-i_{k}+p\right) R_{i_{k}}^{c} / a_{k}\right) .
$$

Note that $\frac{4}{5} \sigma<r_{i}^{c}<\gamma$ for all $i$, and also $\frac{4}{5} \sigma<R_{i_{k}}^{c} / a_{k}<\gamma$ for all $k$. Hence

$$
\begin{array}{ll}
\left(i_{k}+p-j\right) \frac{4}{5} \sigma<\psi_{j}^{1}<\left(i_{k}+p-j\right) \gamma & \left(j<i_{k}\right), \\
\left(j-i_{k}+p\right) \frac{4}{5} \sigma<\psi_{j}^{2}<\left(j-i_{k}+p\right) \gamma & \left(j>i_{k}\right) .
\end{array}
$$

We thus have

$$
\left|h_{i_{k}}\right| \leq\left(\left|W^{\prime \prime}\left(\frac{4}{5} \sigma\right)\right|+2^{2}\left|W^{\prime \prime}\left(2 \frac{4}{5} \sigma\right)\right|+\cdots+i_{c}^{2}\left|W^{\prime \prime}\left(i_{c} \frac{4}{5} \sigma\right)\right|\right) \Delta r_{i_{k}}^{\max } \leq 20 \frac{\Delta r_{i_{k}}^{\max }}{\sigma^{2}}
$$

where $\Delta r_{i_{k}}^{\max }=\max _{j \in\left[i_{k}-i_{c}, i_{k}+i_{c}\right]}\left|r_{j}^{c}-R_{i_{k}}^{c} / a_{k}\right|$. Subtracting (38) from (35) and using Taylor's theorem, we can obtain

$$
\sum_{j=i_{k}-i_{c}}^{i_{k}-1}\left(i_{k}-j\right)^{2} W^{\prime \prime}\left(\left(i_{k}-j\right) \psi\right)\left(R_{i_{k}}-R_{i_{k}}^{c}\right) / a_{k} \leq 20 \frac{\Delta r_{i_{k}}^{\max }}{\sigma^{2}} .
$$


Here $\frac{4}{5} \sigma<\psi=\left(\theta R_{i_{k}}+(1-\theta) R_{i_{k}}^{c}\right) / a_{k}<\gamma($ where $0<\theta<1)$. Noting that

$$
\begin{aligned}
& \sum_{j=i_{k}-i_{c}}^{i_{k}-1}\left(i_{k}-j\right)^{2} W^{\prime \prime}\left(\left(i_{k}-j\right) \psi\right) \\
& \quad \geq W^{\prime \prime}(\gamma)+\sum_{j=2}^{4} j^{2} W^{\prime \prime}\left(j \frac{4}{5} \sigma\right)+\sum_{j=5}^{i_{c}(\delta)} j^{2} W^{\prime \prime}\left(j \frac{4}{5} \sigma\right) \geq 10 / \sigma^{2}
\end{aligned}
$$

and $a_{k} \leq l$, we have

$$
\left|R_{i_{k}}-R_{i_{k}}^{c}\right| \leq 2 l \max _{k} \Delta r_{i_{k}}^{\max }
$$

Now we estimate $\Delta r_{i_{k}}^{\max }$ for the solution $r^{c}$ of (23). From $\frac{4}{5} \sigma<r_{i}^{c}<\gamma \approx 1.12 \sigma$ we immediately have $\Delta r_{i_{k}}^{\max } \leq\left(1.12-\frac{4}{5}\right) \sigma=0.32 \sigma$. However, we can do better. From (25) we have (for all $i \neq j$ )

$$
\begin{aligned}
\left|W^{\prime}\left(r_{i}^{c}\right)-W^{\prime}\left(r_{j}^{c}\right)\right| \leq & \left|W^{\prime}\left(2 \cdot \frac{4}{5} \sigma\right)-W^{\prime}(2 \gamma)\right|+W^{\prime}\left(2 \cdot \frac{4}{5} \sigma\right) \\
& +\left(\left|W^{\prime}\left(3 \cdot \frac{4}{5} \sigma\right)-W^{\prime}(3 \gamma)\right|\right) \\
& +2 W^{\prime}\left(3 \cdot \frac{4}{5} \sigma\right)+\cdots \approx 2 W^{\prime}\left(2 \cdot \frac{4}{5} \sigma\right),
\end{aligned}
$$

because $W^{\prime}(\alpha)$ decreases dramatically as $\alpha$ increases. Noting that $\left|W^{\prime}\left(r_{i}^{c}\right)-W^{\prime}\left(r_{j}^{c}\right)\right|$ $\geq W^{\prime \prime}(\gamma)\left|r_{i}^{c}-r_{j}^{c}\right| \geq 15\left|r_{i}^{c}-r_{j}^{c}\right| / \sigma^{2}$, we obtain $\left|r_{i}^{c}-r_{j}^{c}\right| \leq\left(2 W^{\prime}\left(2 \cdot \frac{4}{5} \sigma\right) / 15\right) \sigma^{2}=\eta \sigma$. Hence,

$$
\Delta r_{i_{k}}^{\max } \leq \eta \sigma
$$

Next we treat (25) more carefully to obtain a much better estimate than (42). Subtracting the equations of (25) for $i=2$ and $i=j+1(j \geq 2)$, we have (noting that $\left.W^{\prime \prime}\left(2 \cdot \frac{4}{5} \sigma\right) 2 W^{\prime}\left(2 \cdot \frac{4}{5} \sigma\right) \leq O\left(3 W^{\prime}\left(3 \cdot \frac{4}{5} \sigma\right)\right)\right)$

$$
\begin{aligned}
& \left|W^{\prime}\left(r_{2}^{c}\right)-W^{\prime}\left(r_{j+1}^{c}\right)\right| \\
& \quad \leq\left|W^{\prime \prime}\left(2 \cdot \frac{4}{5} \sigma\right)\right|\left(\left|r_{1}^{c}-r_{j}^{c}\right|+2\left|r_{2}^{c}-r_{j+1}^{c}\right|+\left|r_{3}^{c}-r_{j+2}^{c}\right|\right)+O\left(3 W^{\prime}\left(3 \cdot \frac{4}{5} \sigma\right)\right) \\
& \quad \leq 2\left|W^{\prime \prime}\left(2 \cdot \frac{4}{5} \sigma\right)\right|\left|r_{2}^{c}-r_{j+1}^{c}\right|+\left|W^{\prime \prime}\left(2 \cdot \frac{4}{5} \sigma\right)\right|\left|r_{3}^{c}-r_{j+2}^{c}\right|+O\left(3 W^{\prime}\left(3 \cdot \frac{4}{5} \sigma\right)\right) .
\end{aligned}
$$

Similarly to the above, we have

$$
\left|r_{2}^{c}-r_{j+1}^{c}\right| \leq O\left(\left|W^{\prime \prime}\left(2 \cdot \frac{4}{5} \sigma\right)\right| / 15\right) \sigma^{2}\left|r_{3}^{c}-r_{j+2}^{c}\right|+O\left(3 W^{\prime}\left(3 \cdot \frac{4}{5} \sigma\right) / 15\right) \sigma^{2} .
$$

Subtracting the equations of (25) for $i=3$ and $i=j+2(j \geq 2)$, we have

$$
\begin{aligned}
& \left|W^{\prime}\left(r_{3}^{c}\right)-W^{\prime}\left(r_{j+2}^{c}\right)\right| \leq\left|W^{\prime \prime}\left(2 \cdot \frac{4}{5} \sigma\right)\right|\left(\left|r_{2}^{c}-r_{j+1}^{c}\right|+2\left|r_{3}^{c}-r_{j+2}^{c}\right|+\left|r_{4}^{c}-r_{j+3}^{c}\right|\right) \\
& \quad+\left|W^{\prime \prime}\left(3 \cdot \frac{4}{5} \sigma\right)\right|\left(\left|r_{1}^{c}-r_{j}^{c}\right|+2\left|r_{2}^{c}-r_{j+1}^{c}\right|+3\left|r_{3}^{c}-r_{j+2}^{c}\right|\right. \\
& \left.\quad+2\left|r_{4}^{c}-r_{j+3}^{c}\right|+\left|r_{5}^{c}-r_{j+4}^{c}\right|\right)+O\left(4 W^{\prime}\left(4 \cdot \frac{4}{5} \sigma\right)\right) .
\end{aligned}
$$


We can verify that

$$
\begin{gathered}
O\left(\left|W^{\prime \prime}\left(2 \cdot \frac{4}{5} \sigma\right)\right| W^{\prime}\left(3 \cdot \frac{4}{5} \sigma\right) / 15\right) \\
O\left(\left|W^{\prime \prime}\left(3 \cdot \frac{4}{5} \sigma\right)\right| W^{\prime}\left(2 \cdot \frac{4}{5} \sigma\right) / 15\right) \leq O\left(4 W^{\prime}\left(4 \cdot \frac{4}{5} \sigma\right)\right) .
\end{gathered}
$$

We thus have

$$
\begin{aligned}
\left|r_{3}^{c}-r_{j+2}^{c}\right| \leq & O\left(\left|W^{\prime \prime}\left(2 \cdot \frac{4}{5} \sigma\right)\right| / 15\right) \sigma^{2}\left|r_{4}^{c}-r_{j+3}^{c}\right| \\
& +O\left(\left|W^{\prime \prime}\left(3 \cdot \frac{4}{5} \sigma\right)\right| / 15\right) \sigma^{2}\left|r_{5}^{c}-r_{j+4}^{c}\right|+O\left(4 W^{\prime}\left(4 \cdot \frac{4}{5} \sigma\right) / 15\right) \sigma^{2}
\end{aligned}
$$

Similarly we can obtain

$$
\begin{aligned}
\left|r_{i}^{c}-r_{j+i-1}^{c}\right| \leq O & \left(\left|W^{\prime \prime}\left(2 \cdot \frac{4}{5} \sigma\right)\right| / 15\right) \sigma^{2}\left|r_{i+1}^{c}-r_{j+i}^{c}\right| \\
& +O\left(\left|W^{\prime \prime}\left(3 \cdot \frac{4}{5} \sigma\right)\right| / 15\right) \sigma^{2}\left|r_{i+2}^{c}-r_{j+i+1}^{c}\right| \\
& +\cdots+O\left(\left|W^{\prime \prime}\left(\left(i \cdot \frac{4}{5} \sigma\right) \mid / 15\right) \sigma^{2}\right| r_{2 i-1}^{c}-r_{j+2 i-2}^{c} \mid\right. \\
& +O\left(i\left|W^{\prime \prime}\left(i \cdot \frac{4}{5} \sigma\right)\right| / 15\right) \sigma^{2}
\end{aligned}
$$

for $1 \leq i \leq i_{c}$. For $i_{c}+1 \leq i \leq\left[\frac{N}{2}\right]$ we have

$$
\begin{aligned}
\left|r_{i}^{c}-r_{j+i-1}^{c}\right| \leq O & \left(\left|W^{\prime \prime}\left(2 \cdot \frac{4}{5} \sigma\right)\right| / 15\right) \sigma^{2}\left|r_{i+1}^{c}-r_{j+i}^{c}\right| \\
& +O\left(\left|W^{\prime \prime}\left(3 \cdot \frac{4}{5} \sigma\right)\right| / 15\right) \sigma^{2}\left|r_{i+2}^{c}-r_{j+i+1}^{c}\right| \\
& +\cdots+O\left(\left|W^{\prime \prime}\left(\left(i_{c} \cdot \frac{4}{5} \sigma\right) \mid / 15\right) \sigma^{2}\right| r_{i+i_{c}-1}^{c}-r_{j+i+i_{c}-2}^{c} \mid\right. \\
& +O\left(i_{c}\left|W^{\prime \prime}\left(i_{c} \cdot \frac{4}{5} \sigma\right)\right| / 15\right) \sigma^{2} .
\end{aligned}
$$

Substituting (44) into (43) and then applying (42), we can dramatically reduce the first term (since $\left|W^{\prime \prime}\left(j \cdot \frac{4}{5} \sigma\right)\right| \ll 1$ for $j \geq 2$ ) and obtain $\left|r_{2}^{c}-r_{j+1}^{c}\right| \leq$ $O\left(3 W^{\prime}\left(3 \cdot \frac{4}{5} \sigma\right) / 15\right) \sigma^{2}$. Similarly, if we substitute (45) for $i=4,5, \ldots$ into (44), we will dramatically reduce the first and the second terms and obtain $\left|r_{3}^{c}-r_{j+2}^{c}\right| \leq$ $O\left(4 W^{\prime}\left(4 \cdot \frac{4}{5} \sigma\right) / 15\right) \sigma^{2}$. Repeating this procedure, we can obtain $\left|r_{i}^{c}-r_{j+i-1}^{c}\right| \leq$ $O\left(i W^{\prime}\left(i \cdot \frac{4}{5} \sigma\right) / 15\right) \sigma^{2}$ for $1 \leq i \leq i_{c}$ and $\left|r_{i}^{c}-r_{j+i-1}^{c}\right| \leq O\left(i_{c} W^{\prime}\left(i_{c} \cdot \frac{4}{5} \sigma\right) / 15\right) \sigma^{2}$ for $i_{c}+1 \leq i \leq\left[\frac{N}{2}\right]$. For $\left[\frac{N}{2}\right] \leq i \leq N$ we can start from $i=N$ and obtain the same estimates accordingly. Therefore, for $k=1$ and $i_{1}-i_{c} \geq i_{c}$

$$
\begin{aligned}
\Delta r_{i_{k}}^{\max } & \leq \max _{i \in\left[i_{k}-i_{c}, i_{k}+i_{c}\right]} \sum_{j=i_{k-1}}^{i_{k}}\left|r_{j}^{c}-r_{i}^{c}\right| / a_{k} \\
& \leq \frac{1}{l} \sum_{j=2}^{i_{c}} j W^{\prime}\left(j \frac{4}{5} \sigma\right) \sigma^{2} / 15+\frac{l-i_{c}}{l} i_{c} W^{\prime}\left(i_{c} \frac{4}{5} \sigma\right) \sigma^{2} \\
& \leq K\left(\frac{\eta}{l}+\frac{l-i_{c}}{l} \delta\right) \sigma .
\end{aligned}
$$


For $k=1$ and $i_{1}-i_{c}<i_{c}$ (noting that $i_{1}=\left[\frac{l}{2}\right]$ ) we have

$$
\begin{aligned}
\Delta r_{i_{k}}^{\max } \leq & \frac{1}{l} \sum_{j=2}^{i_{1}-i_{c}} j\left|W^{\prime}\left(j \frac{4}{5} \sigma\right)\right| \sigma^{2} / 15 \\
& +\frac{l-\left(i_{1}-i_{c}\right)}{l}\left(i_{1}-i_{c}\right)\left|W^{\prime}\left(\left(i_{1}-i_{c}\right) \frac{4}{5} \sigma\right)\right| \sigma^{2} \\
\leq & K\left(\frac{\eta}{l}+\frac{\left[\frac{l}{2}\right]+1+i_{c}}{l}\left|W^{\prime}\left(\left(\left[\frac{l}{2}\right]-i_{c}\right) \frac{4}{5} \sigma\right)\right| \sigma\right) \sigma .
\end{aligned}
$$

For $1<k<\left[\frac{m}{2}\right]$ we can obtain

$$
\Delta r_{i_{k}}^{\max } \leq K \delta \sigma .
$$

For $k \geq\left[\frac{m}{2}\right]$ the result is accordingly the same as (47)-(49). Therefore

$$
\begin{aligned}
& \left\|R_{i_{k}}-R_{i_{k}}^{c}\right\| \\
& \quad \leq\left\{\begin{array}{cl}
K \max \left\{\eta+\left(l-i_{c}\right) \delta, l \delta\right\} \sigma & \text { if }\left[\frac{l}{2}\right]-i_{c} \geq i_{c}, \\
K \max \left\{\eta+\left(\left[\frac{l}{2}\right]+1+i_{c}\right)\left|W^{\prime}\left(\left(\left[\frac{l}{2}\right]-i_{c}\right) \frac{4}{5} \sigma\right)\right| \sigma, l \delta\right\} \sigma & \text { if }\left[\frac{l}{2}\right]-i_{c}<i_{c},
\end{array}\right.
\end{aligned}
$$

where $\|\cdot\|$ can be $\|\cdot\|_{\infty}$ or $\frac{1}{\sqrt{m}}\|\cdot\|_{2}$. Hence, (8) holds due to the first part of (50), (19), and (20). From the definition of $R_{i_{k}}$ and $U_{i_{0}}=0$ we can calculate

$$
U_{i_{1}}=R_{i_{1}}, \quad U_{i_{k}}=\sum_{j=1}^{k} R_{i_{j}}, \text { for } k=1,2, \ldots, m .
$$

Similarly, $u_{i_{k}}^{c}=\sum_{j=1}^{k} R_{i_{j}}^{c}$. Thus the error between $U_{i_{k}}$ and $u_{i_{k}}^{c}$ is

$$
\begin{aligned}
& \left\|U_{i_{k}}-u_{i_{k}}^{c}\right\| \\
& \quad \leq\left\{\begin{array}{cl}
K m \max \left\{\eta+\left(l-i_{c}\right) \delta, l \delta\right\} \sigma & \text { if }\left[\frac{l}{2}\right]-i_{c} \geq i_{c}, \\
K m \max \left\{\eta+\left(\left[\frac{l}{2}\right]+1+i_{c}\right)\left|W^{\prime}\left(\left(\left[\frac{l}{2}\right]-i_{c}\right) \frac{4}{5} \sigma\right)\right| \sigma, l \delta\right\} \sigma & \text { if }\left[\frac{l}{2}\right]-i_{c}<i_{c} .
\end{array}\right.
\end{aligned}
$$

So (9) holds from the first part of (151), (19) and (20). Note that the length of our material problem is of order $O(N \sigma)$. For a material with a finite length we have $m l \sigma=N \sigma=O(1)$.

Combining the error estimate (51) with (77), we obtain the error between the QC approximate solution $U_{i_{k}}$ and the full lattice-scale solution $u_{i_{k}}$ :

$$
\begin{aligned}
& \left\|U_{i_{k}}-u_{i_{k}}\right\|_{\infty} \\
& \quad \leq\left\{\begin{array}{cc}
K(N \sigma)\left(\frac{\eta}{l}+i_{c} \delta\right) & \text { if }\left[\frac{l}{2}\right]-i_{c} \geq i_{c}, \\
K(N \sigma)\left(\frac{\eta}{l}+\frac{\left[\frac{l}{2}\right]+1+i_{c}}{l}\left|W^{\prime}\left(\left(\left[\frac{l}{2}\right]-i_{c}\right) \frac{4}{5} \sigma\right)\right| \sigma+i_{c} \delta\right) & \text { if }\left[\frac{l}{2}\right]-i_{c}<i_{c}
\end{array}\right.
\end{aligned}
$$

The first part of (52) gives (10), using (19) and $l \geq 4 i_{c}$. All notation used in estimates (50)-(52) has been explained in $\S 1$. These results not only prove the error estimates mentioned in the introduction, but also give error estimates for other choices of $l$. Better approximation is expected by using higher order polynomial shape functions in (29). The method we have used to analyze this model may apply to other material particle problems, and our results may also be useful to study dynamic features. 


\section{ACKNOWLEDGMENTS}

The author would like to thank Professor A. Stuart for bringing him into this area and for many stimulating discussions, and to thank Professors K. Cho and H. Gao for many discussions on the physical background.

\section{REFERENCES}

1. J.L. Bassani, V. Vitek and E.S. Alber, Atomic-level elastic properties of interfaces and their relation to continua, Acta Metall. Mater., Vol. 40, 1992, S307-S320.

2. L. Brillouin, Wave Propagation in Periodic Structures, International Series in Physics, McGraw-Hill Book Company, Inc., New York, 1946. MR 8:422d

3. F.C. Frank and J.H. van der Merwe, One-dimensional dislocations. I. Static theory, Proc. R. Soc. London A198, 1949, pp. 205-216.

4. I.A. Kunin, Elastic Media with Microstructure, Vol. I, Springer-Verlag, New York, 1982. MR 84d:73008

5. M. Luskin, On the computation of crystalline microstructure, Acta Numerica, 1996, pp. 191257. MR 99f:73030

6. I.V. Markov, Crystal Growth for Beginners, World Scientific Publishing Co., 1995.

7. E.B. Tadmor, M. Ortiz and R. Phillips, Quasicontinuum analysis of defects in solids, Philosophical Magazine A, Vol. 73, No. 6, 1996, pp. 1529-1563.

Department of Mathematics, The National University of Singapore, 2 Science Drive 2, Singapore 117543

E-mail address: matlinp@math.nus.edu.sg 